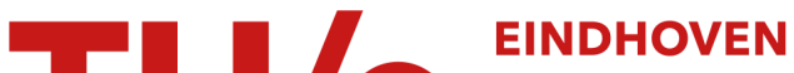 \\ UNIVERSITY OF \\ TECHNOLOGY
}

\section{Control design for an overactuated wheeled mobile robot}

Citation for published version (APA):

Ploeg, J., Vissers, J. P. M., \& Nijmeijer, H. (2006). Control design for an overactuated wheeled mobile robot. In 4th IFAC Symposium on Mechatronic Systems, 12-14 september 2006, Ruprecht-Karls University, Germany, Heidelberg (pp. 127-132)

\section{Document status and date:}

Published: 01/01/2006

\section{Document Version:}

Accepted manuscript including changes made at the peer-review stage

\section{Please check the document version of this publication:}

- A submitted manuscript is the version of the article upon submission and before peer-review. There can be important differences between the submitted version and the official published version of record. People interested in the research are advised to contact the author for the final version of the publication, or visit the $\mathrm{DOI}$ to the publisher's website.

- The final author version and the galley proof are versions of the publication after peer review.

- The final published version features the final layout of the paper including the volume, issue and page numbers.

Link to publication

\section{General rights}

Copyright and moral rights for the publications made accessible in the public portal are retained by the authors and/or other copyright owners and it is a condition of accessing publications that users recognise and abide by the legal requirements associated with these rights.

- Users may download and print one copy of any publication from the public portal for the purpose of private study or research.

- You may not further distribute the material or use it for any profit-making activity or commercial gain

- You may freely distribute the URL identifying the publication in the public portal.

If the publication is distributed under the terms of Article 25fa of the Dutch Copyright Act, indicated by the "Taverne" license above, please follow below link for the End User Agreement:

www.tue.nl/taverne

Take down policy

If you believe that this document breaches copyright please contact us at:

openaccess@tue.nl

providing details and we will investigate your claim. 


\title{
CONTROL DESIGN FOR AN OVERACTUATED WHEELED MOBILE ROBOT
}

\author{
Jeroen Ploeg* John P.M. Vissers ** \\ Henk Nijmeijer ${ }^{* * *}$ \\ * TNO Automotive, P.O. Box 756, 5700 AT Helmond, \\ The Netherlands, Phone: +31 (0)492 566536 , \\ E-mail: jeroen.ploeg@tno.nl \\ ** Vanderlande Industries, Veghel, The Netherlands \\ *** Eindhoven University of Technology, Eindhoven, \\ The Netherlands
}

\begin{abstract}
In order to simulate road vehicles in a hardware-in-the-loop test setup, TNO has developed a wheeled mobile robot with independently driven and steered wheels. This robot is overactuated, i.e. the number of actuators exceeds the number of spatial degrees of freedom to be controlled. A position controller based on feedback linearization is presented. This controller takes the overactuatedness into account by using the so-called multicycle approach, which essentially regards the robot as a combination of independent unicycles. As a result, the robot is position controlled while the redundant actuators are used to compensate for weight transfer during acceleration and cornering. Copyright (C) 2006 IFAC
\end{abstract}

Keywords: Mobile robots, Nonlinear control, Position control, Robotics

\section{INTRODUCTION}

Nowadays, Wheeled Mobile Robots (WMR's) are widely used in industry as transport devices. As such, the main control objective usually is to let a fixed point on the WMR follow a time dependent spatial reference trajectory. TNO Automotive has developed a specific WMR for use in its VeHIL test facility. VeHIL is a hardware-in-theloop testbed for the development of road vehicles equipped with driver assistance functionality based on environment sensors, such as Advanced Cruise Control and Collision Warning. The principle of VeHIL is to simulate only the relative motion of other vehicles with respect to the test vehicle. This allows for efficient, safe and reproducible testing (Gietelink et al., 2004). The neighboring vehicles are simulated by two WMR's, equipped with a dummy vehicle body in order to resemble a real vehicle. Figure 1 shows a photograph of such a
WMR, without body. This VeHIL WMR or Moving Base (MB) differs from most wheeled robots in that it is a high dynamic robot, capable of extreme maneuvers at velocities up to $50 \mathrm{~km} / \mathrm{hr}$, exceeding

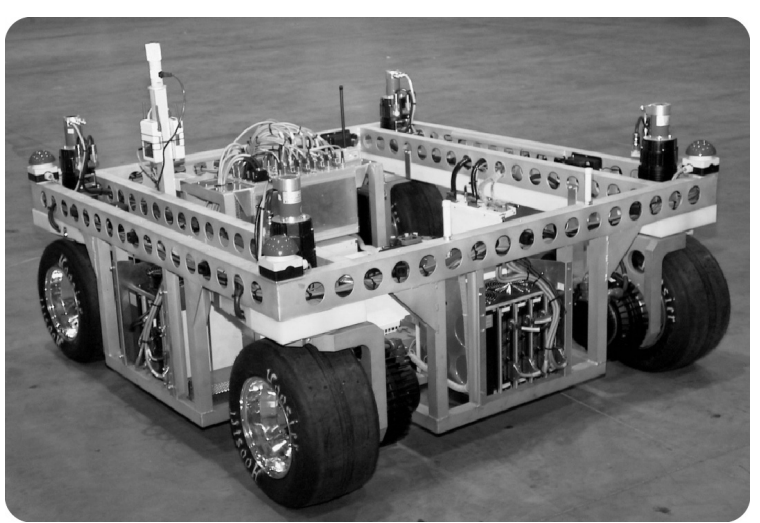

Fig. 1. TNO's mobile robot: the Moving Base 
the handling performance of modern road vehicles (Ploeg et al., 2002).

The control objective of the MB is to let its center follow a reference trajectory $\mathbf{q}_{r e f}$, consisting of the position in the (horizontal) $x, y$-plane and the orientation $\psi$ as a function of the time $t$ :

$$
\mathbf{q}_{r e f}(t)=\left(x_{r e f}(t) y_{r e f}(t) \psi_{r e f}(t)\right)^{\mathrm{T}}
$$

This is achieved by four independently driven and steered wheels. As a consequence, the MB has eight actuators - four driving and four steering motors - whereas the control objective comprises three degrees of freedom only. The MB can therefore be characterized as being overactuated. This paper focuses on the control design for this type of mobile robot.

\section{SPECIFICATIONS}

In order to develop an adequate position controller for the MB, it is important to know its main characteristics. These are shortly summarized here.

The maximum acceleration and deceleration of the $\mathrm{MB}$ is $10 \mathrm{~m} / \mathrm{s}^{2}$ in all directions. The maximum centripetal acceleration is $12 \mathrm{~m} / \mathrm{s}^{2}$. These acceleration levels lead to a considerable weight transfer, given the total mass of the vehicle $(570 \mathrm{~kg})$ and the height of the center of gravity $(0.35 \mathrm{~m})$. The friction force that a tire can deliver, is approximately proportional to the actual vertical load of the tire (Pacejka, 2002), which in turn is determined by the weight transfer. As a consequence, the wheels should be driven by the motors in accordance with their actual vertical loads in order to obtain a maximum performance of the $\mathrm{MB}$ in terms of acceleration and maneuverability. The drive torque distribution across the four wheels should therefore correspond to the actual weight distribution.

The maximum velocity of the $\mathrm{MB}$ is $50 \mathrm{~km} / \mathrm{hr}$ in all directions. It is important for the controller to work well over the whole range from $0-50 \mathrm{~km} / \mathrm{hr}$. Motors capable of steering the wheels in a range of $-350^{\circ}$ till $+350^{\circ}$ are implemented on all four wheels. These large steering angles are necessary in view of the trajectories driven in VeHIL. Table 1 summarizes the main MB specifications.

Table 1. Moving Base specifications

\begin{tabular}{ll}
\hline Vehicle mass (without body) & $570 \mathrm{~kg}$ \\
Wheel base & $1.4 \mathrm{~m}$ \\
Track width & $1.4 \mathrm{~m}$ \\
Center of gravity height & $0.35 \mathrm{~m}$ \\
Maximum velocity & $50 \mathrm{~km} / \mathrm{hr}$ \\
Maximum acceleration & $10 \mathrm{~m} / \mathrm{s}^{2}$ \\
Max. centripetal acceleration & $12 \mathrm{~m} / \mathrm{s}^{2}$ \\
Installed power & $52 \mathrm{~kW}$ \\
Steering angle range & {$\left[-350^{\circ},+350^{\circ}\right]$} \\
\hline
\end{tabular}

\section{CONTROL CONCEPT}

The control method is inspired by the idea presented by Borenstein (Borenstein, 1995), which is to decentralize the tracking problem, the latter being defined as the realization of a desired position and orientation of the vehicle's center, according to (1). To this end, the reference vector $\mathbf{q}_{\text {ref }}$ needs to be converted to reference positions $x_{i j r e f}$ and $y_{\text {ijref }}(i=f($ ront $), r($ ear $), j=l($ eft $), r(i g h t))$ for the four corners where the wheels are attached; refer to figure 2 depicting the $\mathrm{MB}$ co-ordinate systems. The reference orientation angles $\psi_{\text {ijref }}$ of the wheels are calculated using a kinematic approach, i.e. the orientation angles correspond to the direction of the local reference velocities $\dot{x}_{i j r e f}$ and $\dot{y}_{\text {ijref. }}$. As a result, the wheels are oriented such that an instantaneous center of rotation (ICR) is present, being the point where the perpendiculars to the plane of each wheel, drawn from the center of the wheel, are concurrent as illustrated in figure 2. Note that tire slip is thus ignored. Consequently, the reference positions for the MB corners are:

$$
\begin{aligned}
x_{\text {flref }} & =x_{\text {ref }}+L_{d} \cos \left(\psi_{\text {ref }}+\arctan (W / L)\right) \\
y_{\text {flref }} & =y_{\text {ref }}+L_{d} \sin \left(\psi_{\text {ref }}+\arctan (W / L)\right) \\
x_{\text {fref }} & =x_{\text {ref }}+L_{d} \cos \left(\psi_{\text {ref }}-\arctan (W / L)\right) \\
& \ldots
\end{aligned}
$$

where $L$ and $W$ are half the vehicle length and width; $L_{d}=\sqrt{L^{2}+W^{2}}$ is the distance from the center to a corner. According to the kinematic approach, the reference wheel orientation angles $\psi_{\text {ijref }}(i=f, r, j=l, r)$ are:

$$
\psi_{i j r e f}=\arctan \left(\frac{\dot{y}_{i j r e f}}{\dot{x}_{i j r e f}}\right)
$$

where the time derivatives $\dot{x}_{i j r e f}$ and $\dot{y}_{i j r e f}$ are determined by differentiation of equation (2).

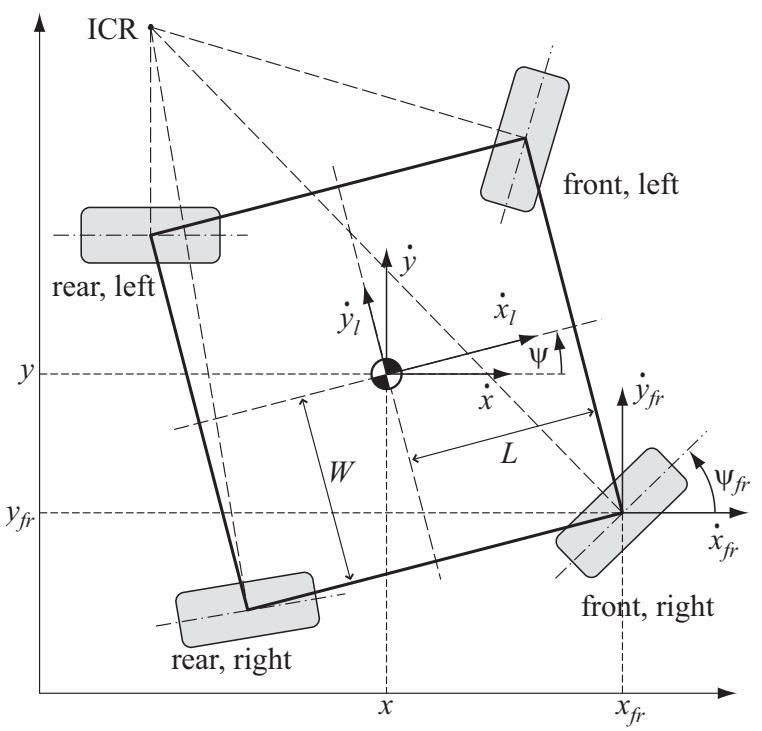

Fig. 2. The Moving Base co-ordinate systems 
The MB is thus regarded as four independent systems, so-called unicycles, being single wheels that can be steered and driven. All four unicycles have their specific reference signal $\mathbf{q}_{\text {ijref }}$ :

$$
\mathbf{q}_{\text {ijref }}(t)=\left(x_{\text {ijref }}(t) y_{\text {ijref }}(t) \psi_{\text {ijref }}(t)\right)^{\mathrm{T}}
$$

So four independent tracking problems effectively remain. As stated in the previous section, weight transfer needs to be taken into account. This can be realized by implementing a mass $m_{i j}$ that varies depending on the acceleration:

$$
\begin{aligned}
& m_{f l}=\frac{m}{4}-\frac{h m}{4 L g} \ddot{x}_{l}-\frac{h m}{4 W g} \ddot{y}_{l} \\
& m_{f r}=\frac{m}{4}-\frac{h m}{4 L g} \ddot{x}_{l}+\frac{h m}{4 W g} \ddot{y}_{l} \\
& m_{r l}=\frac{m}{4}+\frac{h m}{4 L g} \ddot{x}_{l}-\frac{h m}{4 W g} \ddot{y}_{l} \\
& m_{r r}=\frac{m}{4}+\frac{h m}{4 L g} \ddot{x}_{l}+\frac{h m}{4 W g} \ddot{y}_{l}
\end{aligned}
$$

where $m$ is the total mass of the MB, $h$ is the height of the center of gravity, $g$ is the gravitational constant and $\ddot{x}_{l}$ and $\ddot{y}_{l}$ are the accelerations expressed in the local MB co-ordinate system, corresponding to the longitudinal and lateral vehicle acceleration respectively. Note that the latter can be obtained using acceleration measurements or for instance be approximated by taking the reference values following from derivation of equation (2).

The four unicycles now have to be modeled and controlled. To this end, the theory developed in the field of robotics can be applied, as it provides models as well as control methods for unicycles.

\section{UNICYCLE MODELING AND CONTROL}

Before developing a controller for the MB, this section first focuses on the modeling and control design for a unicycle.

\subsection{Modeling}

The unicycle represents a single wheel with a drive motor and a steering motor. If wheel slip is neglected, the robotics theory, based on motion constraints (Campion et al., 1996), can be applied to develop a model. The typical motion constraints for a unicycle are the slip and the roll constraint. The first constraint states that the velocity in lateral direction, i.e. perpendicular to the wheel plane, is zero. The second constraint states that the longitudinal, i.e. forward, velocity of the wheel equals the rotational velocity multiplied by the wheel radius. Using these constraints, combined with dynamic equations describing the drive and steer motor, the following horizontal plane dynamic unicycle model is derived:

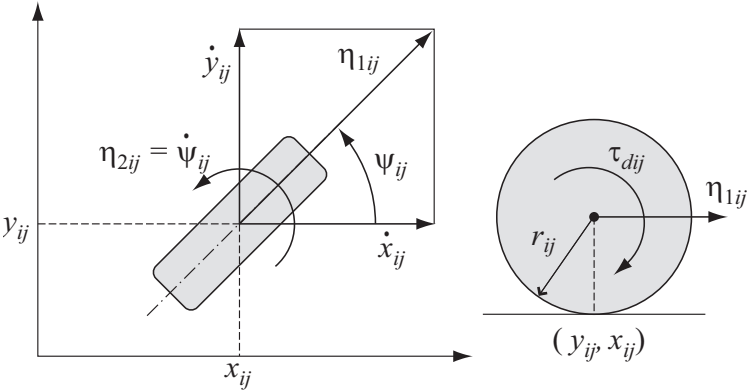

Fig. 3. The unicycle model

$$
\begin{aligned}
\left(\begin{array}{c}
\dot{x}_{i j} \\
\dot{y}_{i j} \\
\dot{\psi}_{i j}
\end{array}\right) & =\left(\begin{array}{c}
\cos \left(\psi_{i j}\right) \eta_{1 i j} \\
\sin \left(\psi_{i j}\right) \eta_{1 i j} \\
\eta_{2 i j}
\end{array}\right) \\
\left(\begin{array}{c}
\dot{\eta}_{1 i j} \\
\dot{\eta}_{2 i j}
\end{array}\right) & =\left(\begin{array}{c}
\frac{r_{i j} \tau_{d i j}}{m_{i j} r_{i j}^{2}+I_{\varphi i j}} \\
\frac{\tau_{s i j}}{I_{\psi i j}}
\end{array}\right)
\end{aligned}
$$

where $x_{i j}, y_{i j}$ and $\psi_{i j}$ are the position and orientation of wheel $i j . \eta_{1 i j}$ and $\eta_{2 i j}$ are the forward and steering velocity respectively. $\tau_{d i j}$ and $\tau_{s i j}$ are the drive and the steer torque, related to the unicycle rotation inertia $I_{\varphi i j}$ and steering inertia $I_{\psi i j} . r_{i j}$ is the wheel radius. Figure 3 illustrates this unicycle model. Finally, if $\tau_{d i j}$ and $\tau_{s i j}$ are chosen as:

$$
\begin{aligned}
\tau_{d i j} & =\frac{m_{i j} r_{i j}^{2}+I_{\varphi i j}}{r_{i j}} \nu_{1 i j} \\
\tau_{s i j} & =I_{\psi i j} \nu_{2 i j}
\end{aligned}
$$

where $\nu_{1 i j}$ and $\nu_{2 i j}$ are new inputs, the dynamic unicycle model can be formulated concisely as:

$$
\begin{aligned}
\dot{\mathbf{q}}_{i j} & =\mathbf{S}\left(\mathbf{q}_{i j}\right) \boldsymbol{\eta}_{i j} \\
\dot{\boldsymbol{\eta}}_{i j} & =\boldsymbol{v}_{i j}
\end{aligned}
$$

with

$$
\mathbf{q}_{i j}=\left(\begin{array}{c}
x_{i j} \\
y_{i j} \\
\psi_{i j}
\end{array}\right) \quad \mathbf{S}\left(\mathbf{q}_{i j}\right)=\left(\begin{array}{cc}
\cos \left(\psi_{i j}\right) & 0 \\
\sin \left(\psi_{i j}\right) & 0 \\
0 & 1
\end{array}\right)
$$

and $\boldsymbol{\eta}_{i j}$ and $\boldsymbol{v}_{i j}$ defined obviously.

\subsection{Control Design}

The unicycle controller will be based on inputoutput linearization by a time-invariant state feedback (Nijmeijer and Schaft, 1990), the advantage of this approach being that linear control techniques can be used for the linearized system. To this end, the system will be linearized first, after which a linear controller will be implemented.

The first step in the linearization procedure is to define an output vector $\mathbf{z}_{1 i j}$ of dimension 2 according to:

$$
\mathbf{z}_{1 i j}=\mathbf{h}\left(\mathbf{q}_{i j}\right)
$$




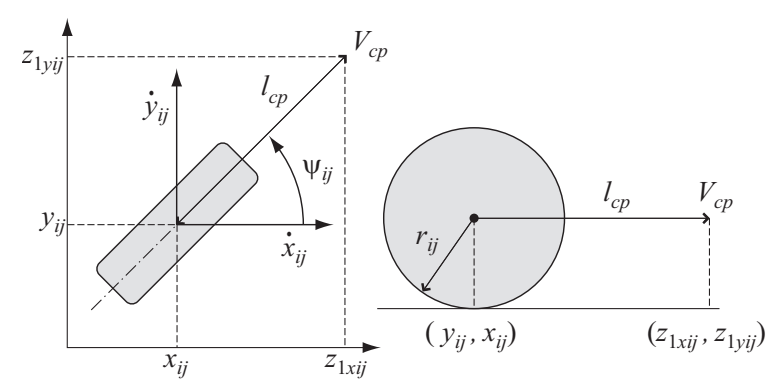

Fig. 4. The virtual control point $V_{c p}$

where $\mathbf{h}: \mathbb{R}^{3} \rightarrow \mathbb{R}^{2}$ is the output function for the unicycle and $\mathbf{z}_{1 i j}$ is the system output to be controlled. As indicated in (9), $\mathbf{z}_{1 i j}$ is constructed from the posture variables $\mathbf{q}_{i j}$. The dimension of $\mathbf{z}_{1 i j}$ is determined by the requirement that the system must be square, i.e. have an equal number of inputs and outputs. A possible choice for the output function $\mathbf{h}$ can be motivated from a physical point of view: if a virtual point $V_{c p}$, lying on a distance $l_{c p}$ in front of the wheel, will be position controlled, then it will be guaranteed that not only the wheel position $x_{i j}, y_{i j}$ but also the wheel orientation $\psi_{i j}$ converge to their reference values as long as a forward velocity exists (and the controlled system is stable). This virtual control point $V_{c p}$ is illustrated in figure 4 . According to this figure, the system output is:

$$
\mathbf{z}_{1 i j}=\left(\begin{array}{c}
z_{1 x i j} \\
z_{1 y i j}
\end{array}\right)=\left(\begin{array}{c}
x_{i j}+l_{c p} \cos \left(\psi_{i j}\right) \\
y_{i j}+l_{c p} \sin \left(\psi_{i j}\right)
\end{array}\right)
$$

The next step comprises differentiation with respect to time of both outputs $z_{1 x i j}$ and $z_{1 y i j}$ until at least one of the inputs $\nu_{1 i j}$ or $\nu_{2 i j}$ appear. The number of differentiations necessary is called the relative degree of each output. Using (8) and (10), the first time derivative $\dot{\mathbf{z}}_{1 i j}$ equals:

$$
\begin{aligned}
\dot{\mathbf{z}}_{1 i j} & =\frac{\partial \mathbf{h}}{\partial \mathbf{q}_{i j}} \dot{\mathbf{q}}_{i j}=\frac{\partial \mathbf{h}}{\partial \mathbf{q}_{i j}} \mathbf{S}\left(\mathbf{q}_{i j}\right) \boldsymbol{\eta}_{i j} \\
& =\mathbf{H}\left(\mathbf{q}_{i j}\right) \boldsymbol{\eta}_{i j}
\end{aligned}
$$

with

$$
\mathbf{H}\left(\mathbf{q}_{i j}\right)=\left(\begin{array}{cc}
\cos \left(\psi_{i j}\right) & -l_{c p} \sin \left(\psi_{i j}\right) \\
\sin \left(\psi_{i j}\right) & l_{c p} \cos \left(\psi_{i j}\right)
\end{array}\right)
$$

Introducing a new state variable $\mathbf{z}_{2 i j}=\dot{\mathbf{z}}_{1 i j}$, the second time derivative equals:

$$
\begin{aligned}
\dot{\mathbf{z}}_{2 i j} & =\mathbf{H}\left(\mathbf{q}_{i j}\right) \dot{\boldsymbol{\eta}}_{i j}+\dot{\mathbf{H}}\left(\mathbf{q}_{i j}\right) \boldsymbol{\eta}_{i j} \\
& =\mathbf{H}\left(\mathbf{q}_{i j}\right) \boldsymbol{v}_{i j}+\mathbf{b}\left(\mathbf{q}_{i j}, \boldsymbol{\eta}_{i j}\right)
\end{aligned}
$$

with

$$
\begin{aligned}
& \mathbf{b}\left(\mathbf{q}_{i j}, \boldsymbol{\eta}_{i j}\right)= \\
& \quad\left(\begin{array}{c}
-\eta_{1 i j} \eta_{2 i j} \sin \left(\psi_{i j}\right)-l_{c p} \eta_{2 i j}^{2} \cos \left(\psi_{i j}\right) \\
\eta_{1 i j} \eta_{2 i j} \cos \left(\psi_{i j}\right)-l_{c p} \eta_{2 i j}^{2} \sin \left(\psi_{i j}\right)
\end{array}\right)
\end{aligned}
$$

Equation (13) clearly shows that the inputs appear in the differential equation after two differentiations. The relative degree of each output therefore equals 2. Note that the determinant $|\mathbf{H}|$ must be nonzero because the inverse $\mathbf{H}^{-1}$ will be applied in the design. As $|\mathbf{H}|=l_{c p}, l_{c p}$ must be nonzero. This requirement can be readily understood because the wheel orientation would be undefined if $V_{c p}$ is located in the wheel center.

The differential equations (11), (13) in fact provide a new description of the linearizable part of the model (8). The order of this subsystem is equal to 4 , corresponding to the total relative degree of both outputs. Note that this is the largest possible subsystem that is feedback linearizable (Bendtsen et al., 2002). Because the order of the original model (8) equals 5 , a subsystem of order 1 remains that cannot be linearized. Let the state of this nonlinear subsystem be denoted by:

$$
z_{3 i j}=k\left(\mathbf{q}_{i j}\right)
$$

where $k: \mathbb{R}^{3} \rightarrow \mathbb{R}$ must be chosen such that the mapping $\left(\mathbf{h}\left(\mathbf{q}_{i j}\right)^{\mathrm{T}} k\left(\mathbf{q}_{i j}\right)^{\mathrm{T}}\right)$ is a diffeomorphism on $\mathbb{R}^{3}$, meaning that the mapping is differentiable and has a differentiable inverse. A possible choice for $k$ is (Canudas de Wit et al., 1996):

$$
k\left(\mathbf{q}_{i j}\right)=\psi_{i j}
$$

Consequently, the time derivative $\dot{z}_{3 i j}$ equals:

$$
\begin{aligned}
\dot{z}_{3 i j} & =\frac{\partial k}{\partial \mathbf{q}_{i j}} \dot{\mathbf{q}}_{i j}=\frac{\partial k}{\partial \mathbf{q}_{i j}} \mathbf{S}\left(\mathbf{q}_{i j}\right) \boldsymbol{\eta}_{i j} \\
& =\frac{\partial k}{\partial \mathbf{q}_{i j}} \mathbf{S}\left(\mathbf{q}_{i j}\right) \mathbf{H}\left(\mathbf{q}_{i j}\right)^{-1} \mathbf{z}_{2 i j} \\
& =\mathbf{f}\left(\mathbf{q}_{i j}\right)^{\mathrm{T}} \mathbf{z}_{2 i j}
\end{aligned}
$$

with

$$
\mathbf{f}\left(\mathbf{q}_{i j}\right)^{\mathrm{T}}=\left(\frac{-\sin \left(\psi_{i j}\right)}{l_{c p}} \frac{\cos \left(\psi_{i j}\right)}{l_{c p}}\right)
$$

With (11), (13) and (17), the unicycle model (8) is now rewritten in the so-called normal form. The actual feedback linearization is obtained by choosing the input vector $\boldsymbol{v}_{i j}$ according to:

$$
\boldsymbol{v}_{i j}=\mathbf{H}\left(\mathbf{q}_{i j}\right)^{-1}\left(\boldsymbol{\omega}_{i j}-\mathbf{b}\left(\mathbf{q}_{i j}, \boldsymbol{\eta}_{i j}\right)\right)
$$

which finally results in the unicycle model:

$$
\begin{aligned}
\dot{\mathbf{z}}_{1 i j} & =\mathbf{z}_{2 i j} \\
\dot{\mathbf{z}}_{2 i j} & =\boldsymbol{\omega}_{i j} \\
\dot{z}_{3 i j} & =\mathbf{f}\left(z_{3 i j}\right)^{\mathrm{T}} \mathbf{z}_{2 i j}
\end{aligned}
$$

with external input $\boldsymbol{\omega}_{i j}$. Note that the argument $\mathbf{q}_{i j}$ of $\mathbf{f}$ has been replaced by $z_{3 i j}$, see (16) and (18). Equation (20) shows that the dynamics of the nonlinear system (8) have now been decomposed into a linear external (input-output) part with states $\mathbf{z}_{1 i j}$ and $\mathbf{z}_{2 i j}$ and a nonlinear internal ("unobservable") part with state $z_{3 i j}$, generally referred to as the internal dynamics.

Tracking behavior of the linear input-output dynamics is obtained by a regular PD-controller with feedforward:

$$
\begin{aligned}
\boldsymbol{\omega}_{i j}=\ddot{\mathbf{z}}_{1 i j r e f}+\mathbf{K}_{v} & \left(\dot{\mathbf{z}}_{1 i j r e f}-\dot{\mathbf{z}}_{1 i j}\right) \\
& +\mathbf{K}_{p}\left(\mathbf{z}_{1 i j r e f}-\mathbf{z}_{1 i j}\right)
\end{aligned}
$$




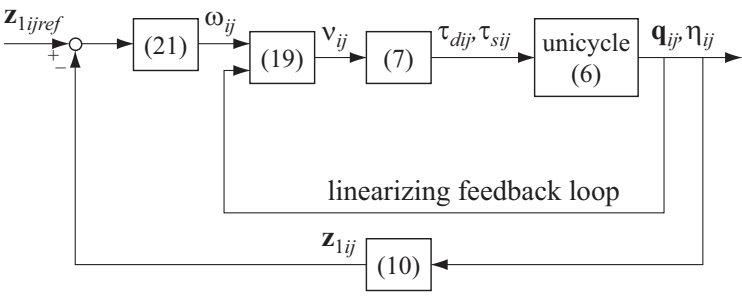

Fig. 5. Block scheme of the controlled unicycle

where $\mathbf{K}_{p}$ and $\mathbf{K}_{v}$ are diagonal $2 \times 2$ matrices containing the proportional and differential gains respectively. In order to obtain equal dynamic behavior in longitudinal and lateral direction, both proportional gains are equal, as are both differential gains. The reference signal $\mathbf{z}_{1 i j r e f}$ is calculated according to (10), using $x_{i j r e f}, y_{i j r e f}$ from (2) and $\psi_{\text {ijref }}$ from (3). Figure 5 shows a block scheme of the controlled unicycle where the numbers refer to the respective equations.

The controller (21) stabilizes the input-output dynamics. The internal dynamics however should also be stable in order to prevent from undesirable phenomena such as very high currents. The internal dynamics are locally stable if the zerodynamics are stable or, in other words, if the nonlinear system is minimum phase. The zerodynamics are the remaining dynamics when the output $\mathbf{z}_{1 i j}$ is identically zero, which implies that its time derivative is zero as well. Implementing this in (20), the zero-dynamics appear to be equal to $\dot{z}_{3 i j}=0$. Hence $z_{3 i j}$ is stable, albeit not asymptotically. Note that in this specific case, the internal dynamics can be shown to be globally stable: from (18) and (20) follows that $z_{3 i j}$ is bounded, provided that $l_{c p}$ is nonzero, because $\dot{\mathbf{z}}_{1 i j}=\mathbf{z}_{2 i j}$ exponentially converges to zero when the controlled system is stable.

\section{MULTICYCLE CONTROL DESIGN}

Having designed a controller for a unicycle, the controller for the entire MB can now be established. As explained in section 3, the MB controller consists of multiple unicycle controllers, one for each wheel corner. It is therefore called the multicycle controller. The wheel corner controllers are almost identical to the unicycle controller as developed in section 4 . One small adaptation however must be made due to the fact that the wheels have to be steered relative to the MB body only.

The unicycle steering torque $\tau_{s i j}$ equals $I_{\psi i j} \ddot{\psi}_{i j}$ for an ideal (frictionless) unicycle. Because however the MB-body also rotates around its vertical axis with angular acceleration $\ddot{\psi}$, the net required steering torque $\tilde{\tau}_{s i j}$ for the multicycle is:

$$
\begin{aligned}
\tilde{\tau}_{s i j} & =I_{\psi i j} \ddot{\delta}_{i j}=I_{\psi i j}\left(\ddot{\psi}_{i j}-\ddot{\psi}\right) \\
& =\tau_{s i j}-I_{\psi i j} \ddot{\psi}
\end{aligned}
$$

where $\delta_{i j}$ is the steering angle, i.e. the wheel orientation with respect to the MB body.. As a consequence, a compensation term $-I_{\psi i j} \ddot{\psi}$ has to be added to $\tau_{s i j}$, which is calculated using (19) and then (7), to obtain the multicycle steering torque. The MB controller is now fully determined.

\section{EXPERIMENTS}

The final step in the control design process entails testing the controller in reality. This means that the controller is implemented on the MB used in VeHIL. The test trajectory that will be shown here is an 'eight-shaped' track in the $x, y$-plane with a fixed orientation as shown in figure 6 . This trajectory comprises two corners with a fixed radius of $3.5 \mathrm{~m}$, illustrating the MB behavior during cornering with a constant lateral tire slip. The maximum centripetal acceleration is $9 \mathrm{~m} / \mathrm{s}^{2}$. The vehicle velocity is limited to $20 \mathrm{~km} / \mathrm{hr}$ (tangential to the track) for safety reasons. A constant orientation $\psi_{\text {ref }}=0$ is chosen in order to illustrate the fact that orientation and position are independent. The trajectory has a non-rolling start, from which the velocity tangential to the track is increased to a prescribed velocity level of $20 \mathrm{~km} / \mathrm{hr}$ with a prescribed acceleration of $5 \mathrm{~m} / \mathrm{s}^{2}$. During the last part of the trajectory, the velocity is decreased to zero with a prescribed acceleration of $-5 \mathrm{~m} / \mathrm{s}^{2}$. The velocity is kept constant between these two phases. At the end, the MB stands still for a few seconds before the data logging is stopped.

The test trajectory is applied to a simulation model of the controlled $\mathrm{MB}$ as well as to the real MB. The simulation model comprises a comprehensive physical model of the MB, implemented together with the multicycle controller in Matlab/Simulink ${ }^{\mathrm{TM}}$. The position and velocity of the MB are estimated by means of a discrete Kalman filter that uses an on-board accelerometer, wheel velocity and steering angle encoders as well as a regularly spaced grid of magnets in the road surface. This observer is implemented in the simulation model as well as in the real MB control system. The estimated position and velocity are used to evaluate the tracking performance. In the real $\mathrm{MB}$, the multicycle controller is implemented in Simulink and then compiled for a Linux target that enables the controller to run in real-time.

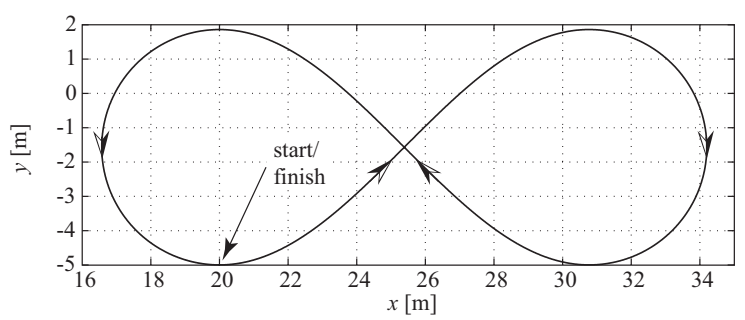

Fig. 6. The reference trajectory in the $x, y$-plane 

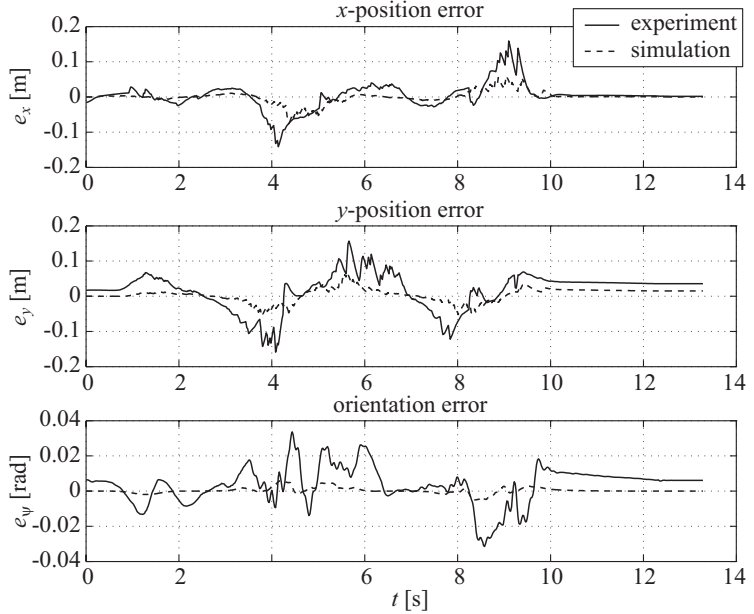

Fig. 7. Simulation and experiment results

Figure 7 shows the results for both the simulation and the practical experiment. This figure displays the $x$-position error $e_{x}=x_{r e f}-x[\mathrm{~m}]$, the $y$ position error $e_{y}=y_{r e f}-y[\mathrm{~m}]$ and the orientation error $e_{\psi}=\psi_{\text {ref }}-\psi[\mathrm{rad}]$ for the $\mathrm{MB}$ center as a function of time. The differences between the practical experiment and the simulation can be assigned to a number of known model uncertainties and simplifications, the most important ones being:

- the tire characteristics are not exactly known;

- the floor in the simulation model is perfectly flat, whereas the test floor is not;

- the friction torque (turn slip) between the tire and the road is ignored in the model.

Although the difference between simulation and reality seems large, it should be noted that figure 7 shows the error signals.

It can be seen that the position error in the practical experiment is reasonable $\left(\left|e_{x}\right|<0.2 \mathrm{~m}\right.$, $\left|e_{y}\right|<0.2 \mathrm{~m}$ and $\left.\left|e_{\psi}\right|<0.04 \mathrm{rad}\right)$, given the high centripetal acceleration during the cornering part of the trajectory. Apart from the non-flat floor and unmodeled friction effects, it might nevertheless be expected that the tracking accuracy is significantly improved when tire slip will be taken into account in the multicycle controller.

Finally, the static errors in the simulation and the practical experiment should be noted. Although for the simulation not clearly visible, none of the errors converges to zero at the end of the simulation. This is a direct consequence of the zero-dynamics as explained in section 4: these dynamics allow for a final nonzero value of each wheel orientation $\psi_{i j}$. Because however $\mathbf{z}_{1 i j}$ does converge to $\mathbf{z}_{1 \text { ijref }}$, the orientation error must be compensated by corresponding errors in the $x$ and $y$-position.

\section{CONCLUSIONS}

Summarizing, the main conclusion is that the multicycle controller successfully controls the wheeled mobile robot, even in high dynamic trajectories. It leads to a relatively simple controller, which can easily be adapted to other platform configurations. In the multicycle approach, the overactuatedness of the robot is employed to optimize the drive torque distribution across the wheels according to the tire characteristics.

A possible improvement of the current multicycle approach lies in the fact that tire slip is neglected on two levels: slip is first ignored on the unicycle control level and then it is ignored again on the multicycle level, namely at the kinematic determination of the reference steering angles. Improvement of the tracking performance accuracy might be expected when taking tire slip into account, as will be the subject of further research.

\section{REFERENCES}

Bendtsen, J.D., P. Anderson and T.S. Pedersen (2002). Robust feedback-linearization based control design for a wheeled mobile robot. In: Proceedings of the 6th International Symposium on Advanced Vehicle Control. Hiroshima, Japan.

Borenstein, J. (1995). Control and kinematic design of multi-degree-of-freedom mobile robots with compliant linkage. IEEE Transactions on Robotics and Automation 11(1), 21-35.

Campion, G., G. Bastin and B. D'Andrea-Novel (1996). Structural properties and classification of kinematic and dynamic models of wheeled mobile robots. IEEE Transactions on Robotics and Automation 12(1), 47-62.

Canudas de Wit, C., B. Siciliano and G. Bastin (1996). Theory of Robot Control. SpringerVerlag. London.

Gietelink, O.J., J. Ploeg, B. de Schutter and M. Verhaegen (2004). VEHIL: A test facility for validation of fault management systems for advanced driver assistance systems. In: Proceedings of the 1st IFAC Symposium on Advances in Automotive Control. Salerno, Italy.

Nijmeijer, H. and A.J. van der Schaft (1990). Nonlinear Dynamical Control Systems. SpringerVerlag. New York.

Pacejka, H.B. (2002). Tire and Vehicle Dynamics. Butterworth-Heinemann. Oxford.

Ploeg, J., A.C.M. van der Knaap and D.J. Verburg (2002). ATS/AGV - design, implementation and evaluation of a high performance AGV. In: Proceedings of the IEEE Intelligent Vehicle Symposium. Versailles, France. 